\title{
Quality assessment of the Ozone_CCI Climate Research Data Package (release 2017): 1. Ground-based validation of total ozone column data products
}

Katerina Garane ${ }^{1}$, Christophe Lerot $^{2}$, Melanie Coldewey-Egbers ${ }^{3}$, Tijl Verhoelst ${ }^{2}$, Irene Zyrichidou $^{1}$, Dimitris S. Balis ${ }^{1}$, Thomas Danckaert ${ }^{2}$, Florence Goutail ${ }^{4}$, Jose Granville ${ }^{2}$, Daan Hubert $^{2}$, Maria Elissavet Koukouli ${ }^{1}$, Arno Keppens ${ }^{2}$, Jean-Christopher Lambert ${ }^{2}$, Diego Loyola $^{3}$, Jean-Pierre Pommereau ${ }^{4}$, Michel Van Roozendael ${ }^{2}$ and Claus Zehner ${ }^{5}$

${ }^{1}$ Laboratory of Atmospheric Physics, Aristotle University of Thessaloniki, Thessaloniki 54124, Greece.

${ }^{2}$ Royal Belgian Institute for Space Aeronomy (BIRA-IASB), 3, Avenue Circulaire, B-1180 Brussels, Belgium.

${ }^{3}$ Deutsches Zentrum für Luft- und Raumfahrt (DLR), Institut für Methodik der Fernerkundung (IMF), 82234 Oberpfaffenhofen, Germany.

${ }^{4}$ LATMOS, CNRS, University Versailles St Quentin, Guyancourt, France

${ }^{5}$ European Space Agency, ESRIN, Frascati, Italy

Correspondence to: Katerina Garane (agarane@auth.gr)

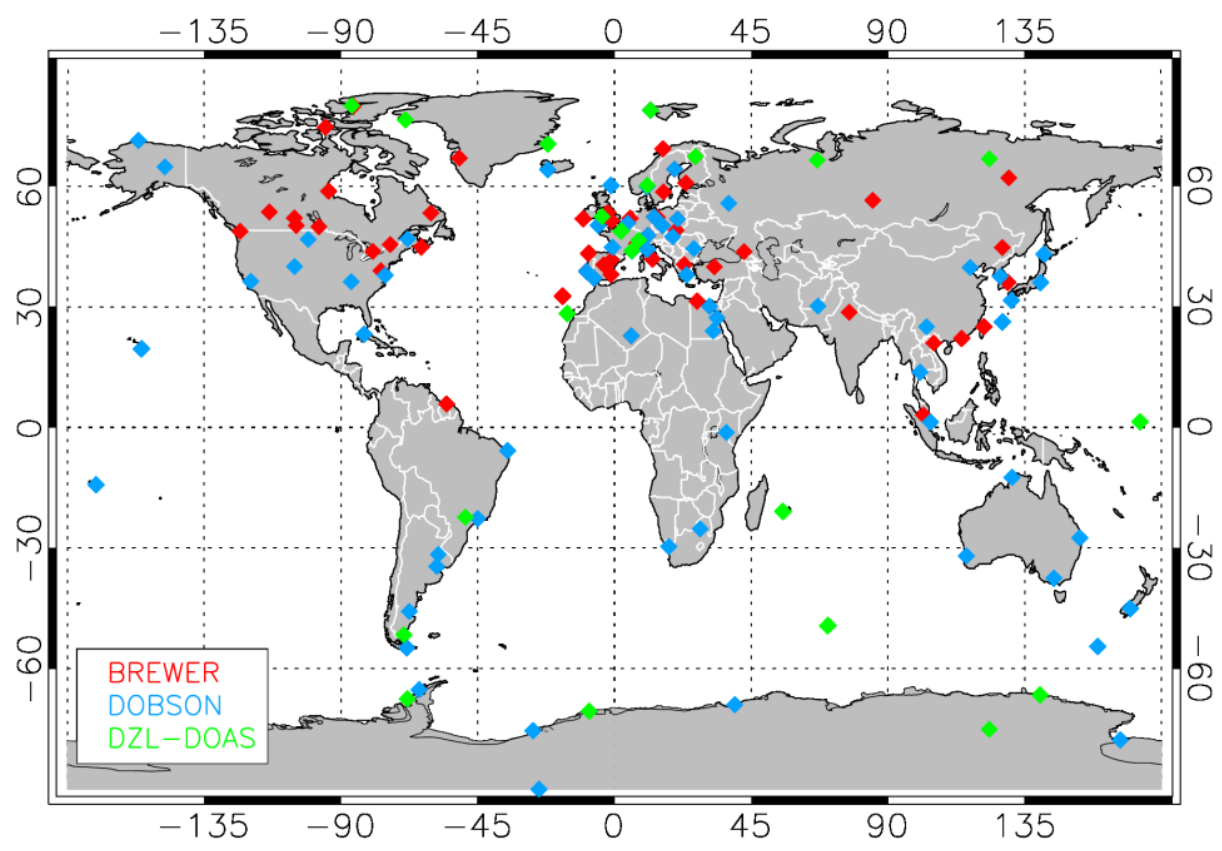

Figure S 1. The locations of the WOUDC/NDACC ground-based instruments reporting total ozone columns used in this study. For further information on these station, refer to Table S 1, Table S 2 and Table S 3. 
Table S 1. The NDACC DOAS UV-Visible instruments selected for this study.

\begin{tabular}{|l|l|l|l|l|l|}
\hline ID & Station & Location & Latitude & Longitude & Instrument \\
\hline 315 & Eureka & Canada & $80.05^{\circ} \mathrm{N}$ & $86.41^{\circ} \mathrm{W}$ & SAOZ \\
\hline 089 & Ny Alesund & Spitsbergen & $78.91^{\circ} \mathrm{N}$ & $11.88^{\circ} \mathrm{E}$ & DOAS \\
\hline 460 & Thule & Western Greenland & $76.53^{\circ} \mathrm{N}$ & $68.74^{\circ} \mathrm{W}$ & SAOZ \\
\hline 459 & Scoresbysund & Eastern Greenland & $70.48^{\circ} \mathrm{N}$ & $21.95^{\circ} \mathrm{W}$ & SAOZ \\
\hline 262 & Sodankylä & Finland & $67.37^{\circ} \mathrm{N}$ & $26.63^{\circ} \mathrm{E}$ & SAOZ \\
\hline 752 & Zhigansk & Eastern Siberia & $66.79^{\circ} \mathrm{N}$ & $123.35^{\circ} \mathrm{E}$ & SAOZ \\
\hline 821 & Salekhard & Western Siberia & $66.50^{\circ} \mathrm{N}$ & $66.70^{\circ} \mathrm{E}$ & SAOZ \\
\hline 658 & Harestua & Norway & $60.20^{\circ} \mathrm{N}$ & $10.80^{\circ} \mathrm{E}$ & DOAS \\
\hline 601 & Aberystwyth & Great Britain & $52.45^{\circ} \mathrm{N}$ & $4.07^{\circ} \mathrm{W}$ & SAOZ \\
\hline 049 & Paris & France & $48.85^{\circ} \mathrm{N}$ & $2.35^{\circ} \mathrm{E}$ & SAOZ \\
\hline & Guyancourt & France & $48.78^{\circ} \mathrm{N}$ & $2.05^{\circ} \mathrm{E}$ & SAOZ \\
\hline 041 & Jungfraujoch & Switzerland & $46.55^{\circ} \mathrm{N}$ & $7.98^{\circ} \mathrm{E}$ & SAOZ \\
\hline 040 & $\begin{array}{l}\text { Observatoire Haute } \\
\text { Province }\end{array}$ & France & $43.94^{\circ} \mathrm{N}$ & $5.71^{\circ} \mathrm{E}$ & SAOZ \\
\hline 300 & Izana & Canaries Island & $28.30^{\circ} \mathrm{N}$ & $15.50^{\circ} \mathrm{W}$ & DOAS \\
\hline 728 & Tarawa & Kiribati & $1.35^{\circ} \mathrm{N}$ & $172.92^{\circ} \mathrm{E}$ & SAOZ \\
\hline 614 & Bauru & Brazil & $22.34^{\circ} \mathrm{S}$ & $49.03^{\circ} \mathrm{W}$ & SAOZ \\
\hline 436 & Reunion & Reunion Island & $20.90^{\circ} \mathrm{S}$ & $55.48^{\circ} \mathrm{E}$ & SAOZ \\
\hline 674 & Kerguelen & Kerguelen Island & $49.35^{\circ} \mathrm{S}$ & $70.26^{\circ} \mathrm{E}$ & SAOZ \\
\hline 817 & Rio Gallegos & Argentina & $51.60^{\circ} \mathrm{S}$ & $69.31^{\circ} \mathrm{W}$ & SAOZ \\
\hline 028 & Dumont d'Urville & Antarctica & $66.67^{\circ} \mathrm{S}$ & $140.02^{\circ} \mathrm{E}$ & SAOZ \\
\hline 709 & Rothera & Antarctic Peninsula & $67.57^{\circ} \mathrm{S}$ & $68.12^{\circ} \mathrm{W}$ & SAOZ \\
\hline 323 & Neumayer & Antarctica & $70.68^{\circ} \mathrm{S}$ & $123.31^{\circ} \mathrm{E}$ & DOAS \\
\hline 641 & Dome Concordia & Antarctica & $75.10^{\circ} \mathrm{S}$ & $123.31^{\circ} \mathrm{E}$ & SAOZ \\
\hline
\end{tabular}

Table S 2. The WOUDC Dobson instruments selected for this study.

\begin{tabular}{|l|l|l|l|l|}
\hline Station ID & Station Name & Station location & Latitude & Longitude \\
\hline 111 & Amundsen Scott & Antarctica & -89.98 & -24.8 \\
\hline 268 & Arrival_Heights & Antarctica & -77.83 & 166.4 \\
\hline 57 & Halley_Bay & Antarctica & -75.52 & -26.73 \\
\hline 101 & Syowa & Antarctica & -69 & 39.58 \\
\hline 232 & VernadskyFaraday & Antarctica & -65.25 & -64.27 \\
\hline 339 & Ushuaia & Argentina & -54.85 & -68.31 \\
\hline 29 & Macquarie_Island & Australia & -54.48 & 158.97 \\
\hline 342 & Comodoro_Rivadavia & Argentina & -45.78 & -67.5 \\
\hline 256 & Lauder & New Zealand & -45.03 & 169.68 \\
\hline 253 & Melbourne & Australia & -37.48 & 144.58 \\
\hline 91 & Buenos-Aires & Argentina & -34.58 & -58.48 \\
\hline 159 & Perth & Australia & -31.95 & 115.85 \\
\hline 343 & Salto & Uruguay & -31.58 & -57.95 \\
\hline 340 & Springbok & South_Africa & -29.67 & 17.9 \\
\hline 27 & Brisbane & Australia & -27.47 & 153.03 \\
\hline 265 & Irene & South_Africa & -25.25 & 28.22 \\
\hline 200 & Cachoeira-Paulista & Brazil & -22.68 & -45 \\
\hline 191 & Samoa & USA & -14.25 & -170.57 \\
\hline 84 & Darwin & Australia & -12.47 & 130.83 \\
\hline 219 & Natal & Brazil & -5.83 & -35.2 \\
\hline 175 & Nairobi & Kenya & -1.27 & 36.8 \\
\hline 214 & Singapore & Singapore & 1.33 & 103.88 \\
\hline 216 & Bangkok & Thailand & 13.73 & 100.57 \\
\hline 31 & Mauna_Loa & USA & 19.53 & -155.58 \\
\hline 2 & Tamanrasset & Algeria & 22.8 & 5.52 \\
\hline & & & & \\
\hline
\end{tabular}




\begin{tabular}{|c|c|c|c|c|}
\hline 311 & Havana & Cuba & 23.17 & -82.33 \\
\hline 245 & Aswan & Egypt & 23.97 & 32.45 \\
\hline 209 & Kunming & China & 25.02 & 102.68 \\
\hline 190 & Naha & Japan & 26.2 & 127.67 \\
\hline 409 & Hurghada & Egypt & 27.25 & 33.72 \\
\hline 152 & Cairo & Egypt & 30.08 & 31.28 \\
\hline 11 & Quetta & Pakistan & 30.18 & 66.95 \\
\hline 7 & Kagoshima & Japan & 31.63 & 130.6 \\
\hline 14 & Tateno & Japan & 36.05 & 140.13 \\
\hline 106 & Nashville & USA & 36.25 & -86.57 \\
\hline 341 & Hanford & USA & 36.32 & -119.63 \\
\hline 213 & El_Arenosillo & Spain & 37.1 & -6.73 \\
\hline 252 & Seoul & Korea & 37.57 & 126.95 \\
\hline 107 & Wallops_Island & USA & 37.87 & -75.52 \\
\hline 293 & Athens & Greece & 38 & 23.7 \\
\hline 82 & Lisbon & Portugal & 38.77 & -9.13 \\
\hline 208 & Shiangher & China & 39.77 & 117 \\
\hline 67 & Boulder & USA & 40.02 & -105.25 \\
\hline 12 & Sapporo & Japan & 43.05 & 141.33 \\
\hline 40 & Haute_Province & France & 43.92 & 5.75 \\
\hline 201 & Sestola & Italy & 44.22 & 10.77 \\
\hline 226 & Bucharest & Romania & 44.48 & 26.13 \\
\hline 419 & Bordeaux & France & 44.81 & -0.56 \\
\hline 19 & Bismarck & USA & 46.77 & -100.75 \\
\hline 35 & Arosa & Switzerland & 46.77 & \begin{tabular}{|l|}
9.67 \\
\end{tabular} \\
\hline 20 & Caribou & USA & 46.87 & -68.02 \\
\hline 100 & Budapest & Hungary & 47.43 & 19.18 \\
\hline 99 & Hohenpeissenberg & Germany & 47.8 & 11.02 \\
\hline 96 & Hradec_Kralove & Czech_Republic & 50.18 & 15.83 \\
\hline 36 & Camborne & UK & 50.22 & -5.32 \\
\hline 53 & Uccle & Belgium & 50.8 & 4.35 \\
\hline 68 & Belsk & Poland & 51.83 & 20.78 \\
\hline 50 & Potsdam & Germany & 52.38 & 13.05 \\
\hline 116 & Moscow & Russia & 55.75 & 37.57 \\
\hline 165 & Oslo & Norway & 59.92 & 10.72 \\
\hline 43 & Lerwick & UK & 60.15 & -1.15 \\
\hline 51 & Reykjavik & Iceland & 64.13 & -21.9 \\
\hline 284 & Vindeln & Sweden & 64.25 & 19.77 \\
\hline 105 & Fairbanks & USA & 64.8 & -147.89 \\
\hline 199 & Barrow & USA & 71.32 & -156.6 \\
\hline 89 & Ny_Alesund & Norway & 78.93 & 11.88 \\
\hline
\end{tabular}

Table S 3. The WOUDC Brewer instruments selected for this study.

\begin{tabular}{|l|l|l|l|l|}
\hline Station ID & Station Name & Station location & Latitude & Longitude \\
\hline 322 & Petaling_Jaya & Malaysia & 3.1 & 101.65 \\
\hline 435 & Paramaribo & Surinam & 5.78 & -55.2 \\
\hline 330 & Hanoi & Vietnam & 21 & 105 \\
\hline 468 & Cape_D'aguilar & Hong Kong & 22.18 & 114.23 \\
\hline 2 & Tamanrasset & Algeria & 22.8 & 5.52 \\
\hline 95 & Taipei & Taiwan & 25.03 & 121.52 \\
\hline 10 & New_Delhi & India & 28.63 & 77.22 \\
\hline 376 & Mrsa_Mtrouh & Egypt & 31.33 & 27.22 \\
\hline 287 & Funchal & Portugal & 32.65 & -17.05 \\
\hline 332 & Pohang & Korea & 36.03 & 129.38 \\
\hline 213 & El_Arenosillo & Spain & 37.1 & -6.73 \\
\hline
\end{tabular}




\begin{tabular}{|c|c|c|c|c|}
\hline 346 & Murcia & Spain & 38 & -1.17 \\
\hline 82 & Lisbon & Portugal & 38.77 & -9.13 \\
\hline 447 & Goddard & USA & 38.99 & -76.83 \\
\hline 348 & Ankara & Turkey & 39.95 & 32.88 \\
\hline 308 & Madrid & Spain & 40.45 & -3.55 \\
\hline 261 & Thessaloniki & Greece & 40.52 & 22.97 \\
\hline 411 & Zaragoza & Spain & 41.658 & -0.944 \\
\hline 305 & Rome_University & Italy & 41.9 & 12.52 \\
\hline 405 & La_Coruna & Spain & 43.33 & -8.5 \\
\hline 282 & Kislovodsk & Russia & 43.73 & 42.66 \\
\hline 65 & Toronto & Canada & 43.78 & -79.47 \\
\hline 326 & Longfenshan & China & 44.75 & 127.6 \\
\hline 321 & Halifax & Canada & 44.9 & -63.5 \\
\hline 319 & Montreal & Canada & 45.47 & -73.75 \\
\hline 479 & Aosta & Italy & 45.71 & 7.33 \\
\hline 301 & Ispra & Italy & 45.8 & 8.63 \\
\hline 35 & Arosa & Switzerland & 46.77 & 9.67 \\
\hline 100 & Budapest & Hungary & 47.43 & 19.18 \\
\hline 99 & Hohenpeissenberg & Germany & 47.8 & 11.02 \\
\hline 290 & Saturna & Canada & 48.78 & -123.13 \\
\hline 331 & Poprad-Ganovce & Slovakia & 49.03 & 20.32 \\
\hline 320 & Winnipeg & Canada & 49.91 & -97.24 \\
\hline 96 & Hradec_Kralove & Czech_Republic & 50.18 & 15.83 \\
\hline 338 & Regina & Canada & 50.21 & -104.67 \\
\hline 53 & Uccle & Belgium & 50.8 & 4.35 \\
\hline 353 & Reading & Great Britain & 51.42 & -0.96 \\
\hline 68 & Belsk & Poland & 51.83 & 20.78 \\
\hline 318 & Valentia & Irland & 51.93 & -10.25 \\
\hline 316 & Debilt & Netherlands & 52 & 5.18 \\
\hline 241 & Saskatoon & Canada & 52.1 & -105.28 \\
\hline 174 & Lindenberg & Germany & 52.22 & 14.12 \\
\hline 50 & Potsdam & Germany & 52.38 & 13.05 \\
\hline 76 & Goose & Canada & 53.32 & -60.38 \\
\hline 352 & Manchester & Great Britain & 53.45 & -2.26 \\
\hline 21 & Edmonton & Canada & 53.57 & -113.52 \\
\hline 481 & Tomsk & Russia & 56.48 & 84.97 \\
\hline 279 & Norkoping & Sweden & 58.58 & 16.12 \\
\hline 77 & Churchill & Canada & 58.75 & -94.07 \\
\hline 404 & Jokioinen & Finland & 60.8 & 23.5 \\
\hline 123 & Yakutsk & Russia & 62.08 & 129.75 \\
\hline 284 & Vindeln & Sweden & 64.25 & 19.77 \\
\hline 267 & Sondrestrom & Greenland & 67 & -50.98 \\
\hline 262 & Sodankyla & Finland & 67.37 & 26.65 \\
\hline 476 & Andoya & Norway & 69.247 & 15.97 \\
\hline 24 & Resolute & Canada & 74.72 & -94.98 \\
\hline 89 & $\mathrm{Ny} \_$Alesund & Norway & 78.93 & 11.88 \\
\hline 315 & Eureka & Canada & 79.89 & -85.93 \\
\hline
\end{tabular}

